\title{
A frequency domain approach to the boundary control problem for parabolic equations
}

\author{
L. Pandolfi \\ Politecnico di Torino, Dipartimento di Matematica \\ Corso Duca degli Abruzzi, 24, 10129 Torino, Italy. \\ Phone: +39-11-5647516. E-mail: lucipan@polito.it
}

\begin{abstract}
The quadratic regulator problem for boundary control systems was studied in many papers, compare Lasiescka and Triggiani (1991) and Bensoussan et al. (1993) for an overview of the existing results. In particular, precise results are available in the case of control systems whose free evolution generates a holomorphic semigroup, due to the high regularity. However, the analysis of the regulator problem was carried out completely in the time domain. Hence, we propose to rederive existing results for parabolic boundary control systems, and standard quadratic cost, following a route which is largely (but not completely) in the frequency domain.
\end{abstract}

\section{Keywords}

Quadratic regulator problem, parabolic equations, frequency domain techniques

\section{INTRODUCTION}

The control system that we are going to study is the system

$\dot{x}=A(x-D u) \quad x(0)=x_{0}$

where as usual $x \in X$, a Hilbert space, and the operator $A$ generates a stable holomorphic semigroup on $X$. The vector $u$ belongs to a Hilbert space $U$ and $D \in \mathcal{L}(U, X)$. Even more, we assume $\operatorname{im} D \subseteq \operatorname{dom}(-A)^{1-\alpha}, 0<\alpha<1$. A crucial consequence of this assumption:

$\|A R(z ; A) D\|=\left\|A(z I-A)^{-1} D\right\|=\leq \frac{1}{|z|^{\alpha}} \quad \forall z \in\{\Re e z>0\}$.

We associate the following cost to the system described by (1)

\footnotetext{
*Paper partially supported by the Italian Ministero della Ricerca Scientifica e Tecnologica within the program of GNAFA-CNR and by HCM network CEC n. ERB-CHRX-CT93-0402.
} 
$J\left(x_{0} ; u\right)=\int_{0}^{+\infty}\left\{\|Q x(t)\|^{2}+\|u(t)\|^{2}\right\} \mathrm{d} t$

where $x(t)=x\left(t ; x_{0}, u\right)$ solves Eq. (1) so that the cost does depend both on the control $u(\cdot)$ and on the initial datum $x_{0}$. We assume that $Q=Q^{*} \in \mathcal{L}(X), Q \geq 0$.

The cost is finite for each square integrable control since we assume that the semigroup generated by the operator $A$ is exponentially stable.

\section{PRELIMINARIES}

In this section we set up the preliminary material and the notations that we use in the present paper.

It is well known that any $L^{2}(\mathbb{R})$-function admits a Laplace transform

$\hat{f}(z)=\int_{0}^{+\infty} e^{-z t} f(t) \mathrm{d} t$

which belongs to the Hardy space $H^{2}$ of the right half plane; i.e. such that

$\sup _{x}\left\{\int_{-\infty}^{+\infty}|f(x+i y)|^{2} \mathrm{~d} y\right\}^{1 / 2}<+\infty$.

Any such function has boundary values on the imaginary axis (defined as non-tangential limits) which identify an $L^{2}(i \mathbb{R})$-function (whose norm is equal to $\left.(2)\right)$. If $f(\cdot)$ is also in $L^{1}(-\infty,+\infty)$ we can define its Fourier transform

$$
F(\omega)=\hat{f}(z)=\int_{-\infty}^{+\infty} e^{-\omega t} f(t) \mathrm{d} t
$$

and it is known that a continuous extension exists to all the square integrable functions, thanks to Parseval equality

$\int_{-\infty}^{+\infty}|f(t)|^{2} \mathrm{~d} t=\frac{1}{2 \pi} \int_{-\infty}^{+\infty}|F(\omega)|^{2} \mathrm{~d} \omega$.

Moreover, $F(\omega)=\hat{f}(i \omega)$. For simplicity we shall always write $f(i \omega)$.

The Fourier transform is a continuous function from $L^{2}(-\infty,+\infty)$ to itself while the Laplace transform is a continuous transformation from $L^{2}(0,+\infty)$ to the Hardy space $H^{2}$ (of the right half plane). We use these facts in order to express the transformations of solutions of Eq. (1).

Let us assume that the input $u(\cdot)$ is both square integrable and $C^{1}$, with square integrable derivative. It is known that this set of functions is dense in $L^{2}(0,+\infty)$. We introduce the function

$\xi(t)=e^{A t} \xi(0)-\int_{0}^{t} e^{A(t-s)} D \dot{u}(s) \mathrm{d} s$

By definition, a solution to Eq. (1) is the function

$x(t)=\xi(t)-D u(t)$ 
and it is possible to prove that

$x(t)=e^{A(t-s)} x_{0}+A \int_{0}^{t} e^{A(t-s)} D u(s) \mathrm{d} s$.

(A formula that can be extended by continuity to any square integrable input $u(\cdot)$, compare Balakrishnan (1978).) Let us take the Laplace transform of the function $\xi(\cdot)$. We have

$\hat{\xi}(i \omega)=R(i \omega ; A) \xi(0)-R(i \omega ; A) D[i \omega \hat{u}(i \omega)-u(0)]$

and $\xi(0)=x_{0}-D u(0)$. from which we obtain

$$
\begin{aligned}
& \hat{x}(i \omega)=R(i \omega ; A) x_{0}-i \omega R(i \omega ; A) D \hat{u}(i \omega)+D \hat{u}(i \omega) \\
& \quad=R(i \omega ; A) x_{0}-A R(i \omega ; A) D \hat{u}(i \omega)=R(i \omega ; A) x_{0}-H(i \omega) \hat{u}(i \omega)
\end{aligned}
$$

Now, this expression was derived under the differentiability assumption on $u(\cdot)$; but, the right hand side depends continuously on $u(\cdot) \in L^{2}(0,+\infty)$ so that we are justified if we assume the expression (3) as the definition of the solution to Eq. (1) for each input $u(\cdot)$ which is simply square integrable.

The assumption that the semigroup be exponentially stable, can be removed.

\section{THE REGULATOR PROBLEM}

After this introduction we study explicitly the system described by

$\dot{\xi}=A \xi-D \dot{u} \quad \xi(0)=\xi_{0}=x(0)-D u(0)$

under the framed assumptions listed in sect. 1. This system is equivalent to (1) only if the control $u(\cdot)$ is differentiable. Consequently, it is not at all obvious that we shall find a minimum for the cost, when using this system.

Fourier transformation gives

$(z I-A)(\hat{x}-D \hat{u})=x_{0}-z D \hat{u}$

i.e.

$\hat{x}=R(z ; A) x_{0}+H(z) \hat{u} \quad H(z)=-A R(z ; A) D$

Parseval equality implies that we must minimize

$\hat{J}\left(x_{0}, \hat{u}\right)=\int_{-\infty}^{+\infty}\left\{|Q \hat{x}(i \omega)|^{2}+|\hat{u}(i \omega)|^{2}\right\} \mathrm{d} \omega$

on the solutions to Eq. (6). We noted already that it is conceivable that we lost the optimal control, if the optimal control is not differentiable. But, in fact, we see directly that Eq. (3) makes sense for every square integrable control $\hat{u}(\cdot)$ and that $\hat{x}(\cdot)$ is a continuous 
function of $\hat{u}(\cdot)$ (in the $L^{2}$ norms). Hence, the minimum of the cost defined in (8) along the solutions of $(3)$ exists and is unique for each given $x_{0}$.

We can characterize the optimal control $u^{+}\left(\cdot ; x_{0}\right)$ (and the optimal state $x^{+}\left(\cdot ; x_{0}\right)$ ) in the frequency domain in two ways, both useful. The first is easier:

$\left\langle\hat{v}, \hat{u}^{+}+H^{*} Q \hat{x}^{+}\right\rangle=0 \quad \forall \hat{v} \in H^{2}$.

This condition is obtained by computing the derivative at $\rho=0$ of the function $\rho \rightarrow$ $\hat{J}\left(x_{0}, \hat{u}^{+}+\rho \hat{v}\right)$ for any $\hat{v} \in H^{2}$. Hence, we find that

$\hat{u}^{+}=-P_{+} M_{H \cdot Q} \hat{x}^{+}$

where $P_{+}$denotes the projection of $L^{2}(i \mathbb{R})$ onto $H^{2}$ and $M_{H^{*} Q}$ is the multiplication by $H^{*} Q$.

A second expression for the optimal control is more artificial. We extend the control $u(\cdot)$ to the negative axis. Let us assume for the moment that the extended function is differentiable. We introduce the function

$\tilde{\xi}(t)=\left\{\begin{array}{ll}e^{A t}\left\{\xi_{0}+\int_{-\infty}^{0} e^{-A s} D \dot{u}(s) \mathrm{d} s\right\} & \text { if } t>0 \\ 0 & \text { if } t<0\end{array}-\int_{-\infty}^{t} e^{A(t-s)} D \dot{u}(s) \mathrm{d} s\right.$.

Moreover, we define $\tilde{x}(\cdot)=\tilde{\xi}(\cdot)+D u(\cdot)$ so that

$\tilde{x}(t)= \begin{cases}x\left(t ; x_{0}, u\right) & \text { if } t>0 \\ -\int_{-\infty}^{t} e^{A(t-s)} D \dot{u}(s) d s+D u(t) & \text { if } t<0\end{cases}$

and, we define $\tilde{J}\left(x_{0} ; u\right)=\int_{-\infty}^{+\infty}\left\{\langle\tilde{x}, Q \tilde{x}\rangle+|u(t)|^{2}\right\} \mathrm{d} t$. We note that neither $\tilde{\xi}(\cdot)$ nor $\tilde{x}(\cdot)$ are continuous at $t=0$; But, the transformations from $u(\cdot)$ to $\tilde{\xi}(\cdot)$ and to $\tilde{x}(\cdot)$ are continuous transformations from $L^{2}(-\infty,+\infty ; U)$ to $L^{2}(-\infty,+\infty ; X)$.

As $\tilde{x}(t)=x(t)$ for $t>0$ then $J\left(x_{0}, u\right) \leq \tilde{J}\left(x_{0}, u\right)$ and equality holds if $u(\cdot)$ is zero for negative times. Hence: $\inf _{u} \tilde{J}\left(x_{0} ; u\right)=\inf _{u} J\left(x_{0} ; u\right)$. We see that if we compute the function which minimize the cost $\tilde{J}\left(x_{0} ; u\right)$ we find the optimal control for the original regulator problem (1), (2): The artificial extension of the control $u(\cdot)$ to the negative axis is not going to change either the optimal control or the value of the minimum.

We take the Fourier transforms and we get the following form for of $\hat{\tilde{\xi}}(\cdot)$, when $u(\cdot) \in$ $C^{1}(-\infty ;+\infty)$ :

$\hat{\tilde{\xi}}(i \omega)=R(i \omega ; A)\left\{\xi_{0}+\int_{-\infty}^{0} e^{-A s} D \dot{u}(s) \mathrm{d} s\right\}-i \omega R(i \omega, A) D \hat{u}(i \omega)$

$=R(i \omega ; A)\left[\xi_{0}+D u(0)\right]-D \hat{u}(i \omega)+H(i \omega) \hat{u}(i \omega)+A R(i \omega ; A) \nu$

and

$\nu=\int_{-\infty}^{0} e^{-A s} D u(s) \mathrm{d} s=\frac{1}{2 \pi} \int_{-\infty}^{+\infty} R(i \omega ; A) D \hat{u}(i \omega) \mathrm{d} \omega \in \operatorname{dom} A^{\gamma} \quad \gamma<1 / 2+\alpha$. 
Consequently, we add $D \hat{u}$ and we get

$\hat{\tilde{x}}(i \omega)=R(i \omega, A) x_{0}+H(i \omega) \hat{u}(i \omega)+A R(i \omega ; A) \nu$.

Now we minimize in the frequency domain the functional $\tilde{J}$. We differentiate with respect to $\rho$ the expression

$\tilde{J}\left(x_{0}, \hat{u}^{+}+\rho \hat{v}\right)=$

$=(1 / 2 \pi) \int_{-\infty}^{+\infty}\left\{\left\langle\hat{x}^{+}+\rho H \hat{v}+\rho \Lambda \hat{v}, Q\left[\hat{x}^{+}+\rho H \hat{v}+\rho \Lambda \hat{v}\right]\right\rangle+\left\langle\hat{u}^{+}+\rho \hat{v}, \hat{u}^{+}+\rho \hat{v}\right\rangle\right\} \mathrm{d} \omega$

where

$\Lambda \hat{v}=A R(i \omega ; A) \int_{-\infty}^{0} e^{-A s} D v(s) \mathrm{d} s$

The derivative above must be zero for each $\hat{v} \in L^{2}(i \mathbb{R} ; U)$. Consequently,

$\hat{u}^{+}=-H^{*} Q \hat{\tilde{x}}^{+}-\Lambda^{*} Q \hat{\tilde{x}}^{+}$

The projection operator is now replaced by the subtraction of $-\Lambda^{*} Q \hat{\tilde{x}}^{+}$(and, we recall, $\left.\tilde{x}^{+}(\cdot)=\tilde{x}(\cdot)\right)$.

A point should be noted: we are making these computations in the frequency domain; but, the operator $\Lambda$ acts on the time function $v(\cdot)$. We should take this into account in the computation of the of the adjoint of $\Lambda$. Standard calculations give $\Lambda^{*}$ :

$\Lambda^{*} \hat{z}=D^{*} A^{*} R^{*}(i \omega ; A) \int_{-\infty}^{+\infty} R^{*}(i s ; A) \hat{z}(i s) \mathrm{d} s,\left\|\Lambda^{*} \hat{z}\right\|=O\left(1 / \omega^{1-\alpha}\right)$

Now we have:

Theorem 1 The function $t \rightarrow x^{+}(t)$ is continuous for each $t \geq 0$ and each $x_{0}$.

Proof. The Fourier transform of $t \rightarrow x^{+}(t)$ is the function

$\hat{x}(i \omega)=R(i \omega ; A) x_{0}+H(i \omega) \hat{u}^{+}(i \omega)$

The inverse transform of $R(i \omega ; A) x_{0}$ is the function $e^{A t} x_{0}$ for $t \geq 0$ while it is zero for $t<0$ : it is a continuous function for $t \geq 0$.

It is known that the inverse Fourier transform of an $L^{1}(i \mathbb{R})$ is continuous on $\mathbb{R}$ Hence, if we can prove that $H(i \omega) \hat{u}(i \omega)$ is an integrable function then the inverse Laplace transform of $H(i \omega) \hat{u}^{+}(i \omega)$ is even continuous on $\mathbb{R}$ The following bootstrap argument shows that $H(i \omega) \hat{u}^{+}(i \omega)$ is integrable by proving that $\hat{u}(i \omega)=O\left(1 / \omega^{1-\alpha+c}\right)$ for large $|\omega|$.

A direct observation of the expression (11) for $\hat{\tilde{x}}(i \omega)$ shows that $\hat{\tilde{x}}(i \omega)=O\left(1 / \omega^{\sigma}\right)$ for some positive $\sigma, 0<\sigma<\min \{\alpha,(3 / 2)-\alpha$. 
We use this observation in the expression (13) for $\hat{u}(i \omega)=-H^{*} Q \hat{\tilde{x}}^{+}-\Lambda^{*} Q \hat{\tilde{x}}^{+}$and we find that the first addendum is of the order $O\left(1 / \omega^{\alpha+\sigma}\right)$ and the second addendum is of the order $O\left(1 / \omega^{\alpha+\sigma-\epsilon}\right)$. In fact,

$$
\left(\Lambda^{*} \hat{\tilde{x}}\right)(i \omega)=D^{*}(-A)^{* \alpha}(-A)^{* 1-\alpha-\sigma+\varepsilon} R^{*}(i \omega) \int_{-\infty}^{+\infty}(-A)^{* \sigma-\epsilon} R^{*}(i s ; A) O\left(1 / s^{\sigma}\right) \mathrm{d} s
$$

The integral is convergent and the factor in front of it is of the order of $1 / \omega^{\alpha+\sigma-\epsilon}$. Hence, this is also the order of $\hat{u}(i \omega)$ for $|\omega| \rightarrow+\infty$.

In the case that $\alpha+\sigma>1$ we are done; otherwise we repeat this procedure. We replace again in the expression (11) for $\hat{\tilde{x}}^{+}(i \omega)$ and we find that

$$
\hat{\tilde{x}}^{+}(i \omega)=O(1 / \omega)+O\left(1 / \omega^{2 \alpha+\sigma-\epsilon}\right)+O\left(1 / \omega^{\alpha+\omega-2 \epsilon}\right):
$$

we gained $\alpha-2 \epsilon$ of regularity above the previous estimate as long as $\alpha+\sigma-2 \epsilon<0$.

If we insert this further information in the expression (13) we see that $\hat{u}^{+}(i \omega)=$ $O\left(1 / \omega^{\alpha+\epsilon+(\alpha+\sigma-2 \epsilon)}\right)$.

We iterate these steps till we have

$$
\hat{u}(i \omega)=O\left(1 / \omega^{1-\alpha+\epsilon}\right)
$$

and consequently $\hat{x}(i \omega)$ is the sum of $R(i \omega ; A) x_{0}$ (whose inverse Fourier transformation is continuous on $t \geq 0$ ) and of a function which is continuous on the whole real axis.

Analogously,

Theorem 2 The function $t \rightarrow u^{+}\left(t ; x_{0}\right)$ is a continuous function of $t$ for $t \geq 0$ and moreover $x_{0} \rightarrow u^{+}\left(t ; x_{0}\right)$ is continuous for each $t \geq 0$; the function $x_{0} \rightarrow u^{+}\left(\cdot ; x_{0}\right)$ is continuous from $X$ to $L^{2}(0,+\infty)$.

Proof. We replace $\hat{u}^{+}(i \omega)$ in the the expression (11) of $\hat{\tilde{x}}(i \omega)$; then we replace the resulting formula in the expression (13) of the optimal control. We note that when $v=u^{+}$then $A R(i \omega ; A) \nu=\Lambda \hat{u}^{+}$, compare (12). Hence,

$\hat{u}^{+}(i \omega)=-\left\{I+(H+\Lambda)^{*} Q(H+\Lambda)\right\}^{-1}\left\{H^{*}+\Lambda^{*}\right\} Q R(i \omega) x_{0}$.

The first brace is bounded for large $|\omega|$ and the second is the order of $1 / \omega^{\alpha}$ so that $\omega \rightarrow \hat{u}^{+}(i \omega)$ is integrable, since $R(i \omega ; A)=O(1 / \omega)$ : the optimal control is a time continuous function. Moreover, the expression above depends continuously, as an $L^{1}(-\infty,+\infty)$ function, on $x_{0}$. This shows that $x_{0} \rightarrow u^{+}\left(t ; x_{0}\right)$ is continuous for each time $t \geq 0$.

The next crucial step is the following result:

Theorem 3 The transformation $\left(t, x_{0}\right) \rightarrow x^{+}\left(t ; x_{0}\right)$ is a $C_{0}$-semigroup.

Proof. We know that $t \rightarrow x\left(t ; x_{0}\right)$ is a continuous function from $[0,+\infty)$ to $X$ and that $x_{0} \rightarrow x\left(t ; x_{0}\right)$ is continuous from $X$ to $X$ (compare previous Lemma). 
The semigroup property is a consequence of the causality of the problem, and can be proved with a time domain argument for example as in Lasiecka, Lukes, Pandolfi, (1995).

Let use denote $A_{+}$the infinitesimal generator of the semigroup $T^{+}(t) x_{0}=x^{+}\left(t ; x_{0}\right)$. We note that $A_{+}$generates an exponentially stable semigroup because we know the function $t \rightarrow x^{+}\left(t ; x_{0}\right)$ is square integrable for each $x_{0}$. Hence, exponential stability follows from Datko (1970).

It is also possible to study further regularity property of the optimal control. We already noted continuity. Moreover, we can prove

Theorem 4 The optimal control belongs to $W^{k, 2}$ if $x_{0} \in \operatorname{dom}\left(A_{+}\right)^{k+1}$.

Proof. We present first a different proof of the continuity of the optimal control, which however requires the further condition $x_{0} \in \operatorname{dom} A_{+}$. We proved that

$\hat{u}^{+}(i \omega)=P_{+} M_{H^{*} Q} \hat{x}^{+}(i \omega)=P_{+}\left(H^{*}(i \omega) Q R\left(i \omega ; A_{+}\right) x_{0}\right)$

so that $t \rightarrow u^{+}(t)$ is the restriction to $t \geq 0$ of the inverse Fourier transform of $M_{H^{*} Q} x^{+}$. We prove that this inverse Fourier transform is continuous on $\mathbb{R}_{4}$. We note that the inverse Fourier transform of $x_{0} /(1+i \omega)$ is zero for $t<0$ and it is $e^{-t} x_{0}$ for $t \geq 0$ : its restriction to $\mathbb{R}_{+}$is continuous. Now,

$$
\begin{aligned}
& H^{*} Q\left\{R\left(i \omega, A_{+}\right) x_{0}-\frac{1}{i \omega+1} x_{0}\right\}=H^{*} Q\left(A_{+}+I\right) \frac{R\left(i \omega, A_{+}\right)}{i \omega+1} x_{0} \\
& =\left[\frac{1}{i \omega+1} H^{*}(i \omega)\right]\left[Q R\left(i \omega, A_{+}\right)\left(A_{+}+I\right) x_{0}\right]
\end{aligned}
$$

if $x_{0} \in \operatorname{dom} A_{+}$. The second bracket is bounded on the imaginary axis since $A_{+}$generates an exponentially stable semigroup. The first bracket is $O\left(1 / \omega^{1+\alpha}\right)$ is integrable. Hence, the inverse Fourier transform is continuous.

We can iterate this procedure and we find that the optimal control belongs to $W^{k, 2}$ if $x_{0} \in \operatorname{dom}\left(A_{+}\right)^{k+1}$. We show this for the case $k=1$. In this case $x_{0} \in \operatorname{dom} A_{+}^{2}$ and we consider

$$
\begin{aligned}
& H^{*} Q\left\{R\left(i \omega ; A_{+}\right) x_{0}-\frac{1}{i \omega+1} x_{0}-\frac{\left(A_{+}+I\right)}{(i \omega+1)^{2}} x_{0}\right\} \\
& =\frac{1}{i \omega+1} H^{*}(i \omega) Q\left(A_{+}+I\right)\left\{R\left(i \omega ; A_{+}\right)-\frac{1}{i \omega+1} x_{0}\right\} \\
& \frac{1}{(i \omega+1)^{2}} H^{*}(i \omega) Q R\left(i \omega ; A_{+}\right)\left(A_{+}+I\right)^{2} x_{0}=O\left(1 / \omega^{2}\right)
\end{aligned}
$$

i.e. its inverse transform belongs to $W^{1,2}(-\infty,+\infty ; U)$. As both the inverse transforms of $\frac{x_{0}}{i \omega+1}$ and of $\frac{\left(A_{+}+I\right) x_{0}}{(i \omega+1)^{2}}$ belong to $W^{1,2}(0,+\infty ; U)$ we have the result.

Now we consider the value of the optimal cost:

$$
\hat{J}\left(x_{0} ; u^{+}\right)=\left\langle R(i \omega ; A) x_{0}+H \hat{u}^{+}, Q \hat{x}^{+}\right\rangle_{L^{2}}+\langle\hat{u}, \hat{u}\rangle_{L^{2}}
$$


$=\left\langle R(i \omega ; A) x_{0}, Q \hat{x}^{+}\right\rangle+\left\{\left\langle\hat{u}^{+}, H^{*} Q \hat{x}^{+}+\hat{u}^{+}\right\rangle\right\}=\left\langle R(i \omega ; A) x_{0}, Q \hat{x}^{+}\right\rangle$.

In fact, the inner product $\left\langle\hat{u}^{+}, H^{*} Q \hat{x}^{+}+\hat{u}^{+}\right\rangle$is zero, compare Eq. (9). Consequently

$$
\begin{aligned}
& J\left(x_{0}, u^{+}\right)=\left\langle x_{0}, P x_{0}\right\rangle=(1 / 2 \pi)\left\langle x_{0}, \int_{-\infty}^{+\infty} R^{*}(i \omega ; A) Q \hat{x}^{+}\left(i \omega ; x_{0}\right) \mathrm{d} \omega\right\rangle \\
& =\left\langle x_{0}, \int_{0}^{+\infty} e^{A^{*} t} Q e^{A^{+} t} x_{0} \mathrm{~d} t\right\rangle .
\end{aligned}
$$

It follows from these inequalities that $\operatorname{im} P \subseteq \operatorname{dom}\left(-A^{*}\right)^{1-\epsilon}$ for each $\epsilon>0$ (compare Lasiecka, Triggiani (1991)). In particular, $D^{*} A^{*} P$ admits a continuous extension to $X$. We proved already that $t \rightarrow u^{+}\left(t ; x_{0}\right)$ is continuous if $x_{0} \in \operatorname{dom} A^{+}$. Hence we can compute $u^{+}\left(0 ; x_{0}\right)$ which is the value of the inverse Fourier transform at 0 :

$$
\begin{aligned}
& u^{+}\left(0 ; x_{0}\right)=-(1 / 2 \pi) \int_{-\infty}^{+\infty} H^{*}(i \omega) Q \hat{x}^{+}\left(i \omega ; x_{0}\right) \mathrm{d} \omega \\
& =-D^{*} A^{*}\left\{\int_{-\infty}^{+\infty} R^{*}(i \omega ; A) Q \hat{x}\left(i \omega ; x_{0}\right) \mathrm{d} \omega\right\}=-D^{*} A^{*} P x_{0} .
\end{aligned}
$$

(We can use the formula for the inverse Fourier Transform since the integrand belongs to $\left.L^{1}(i \mathbb{R})\right)$. The previous relation was proved under the assumption that $x_{0} \in \operatorname{dom} A^{+}$. We saw that $x_{0} \rightarrow u^{+}\left(0 ; x_{0}\right)$ is continuous on $X$ so that $x_{0} \rightarrow D^{*} A^{*} P x_{0}$ admits a continuous extension to $X$, i.e. equality (15) holds on $X$.

In particular, the function $t \rightarrow u^{+}\left(t ; x_{0}\right)$ is bounded on $[0,+\infty)$.

Now,

Theorem 5 If $x \in \operatorname{dom} A_{+}$then $P x \in \operatorname{dom} A^{*}$; if $x \in \operatorname{dom} A$ then $P x \in \operatorname{dom} A^{*}$.

Proof. We show that $y \rightarrow\langle P x, A y\rangle$ is continuous on $X$ for each $x \in \operatorname{dom} A^{+}$. In fact, we make use of Parseval equality and we see that

$$
\begin{aligned}
& \langle P x, A y\rangle=(1 / 2 \pi) \int_{-\infty}^{+\infty}\left\langle Q R\left(i \omega ; A^{+}\right) x, A R(i \omega ; A) y\right\rangle \mathrm{d} \omega=\int_{0}^{+\infty}\left\langle Q e^{A^{+} s} x, A e^{A s} y\right\rangle \mathrm{d} s \\
& =-\langle Q x, y\rangle-\int_{0}^{+\infty}\left\langle Q e^{A^{+} s} A^{+} x, e^{A s} y\right\rangle \mathrm{d} s
\end{aligned}
$$

a continuous function of $y$.

In order to see the second property, we note that

$$
\hat{x}^{+}(i \omega)=R(i \omega ; A) x_{0}+H(i \omega) \hat{u}^{+}(i \omega)=R(i \omega ; A) A^{-1} A x_{0}=O\left(1 / \omega^{2}\right)+O\left(1 / \omega^{\alpha}\right) \hat{u}^{+}(i \omega) .
$$

Hence,

$$
A^{*} P x_{0}=\int_{-\infty}^{+\infty} A^{*} R^{*}(i \omega ; A) x^{+}\left(i \omega ; x_{0}\right)
$$

is expressed by a convergent integral. 


\section{Moreover,}

\section{Theorem 6 The operator $A^{+}$generates a holomorphic semigroup.}

Proof. The system is causal. This implies that $u^{+}\left(s+\tau ; x_{0}\right)=u^{+}\left(s ; x^{+}\left(\tau ; x_{0}\right)\right) \forall s, \tau \geq 0$.

We put $s=0$ and we get:

$$
u^{+}\left(\tau ; x_{0}\right)=u^{+}\left(0 ; x^{+}\left(\tau, x_{0}\right)\right)=-D^{*} A^{*} P x^{+}\left(\tau, x_{0}\right) \quad \forall x_{0} \in \operatorname{dom} A^{+} .
$$

If $x_{0} \in \operatorname{dom} A^{+}$then $t \rightarrow x^{+}\left(t ; x_{0}\right)$ is differentiable so that

$$
\dot{x}^{+}(t)=A\left[I-D D^{*} A^{*} P\right] x^{+}(t)
$$

i.e. $\operatorname{dom} A^{+}=\left\{x,\left[I-D D^{*} A^{*} P\right] x \in \operatorname{dom} A\right\} \quad A^{+} x=A\left[I-D D^{*} A^{*} P\right] x$. As in Lasiecka, Triggiani (1991) this implies that

$$
\left(z I-A^{+}\right)^{-1}=\left[z I-A\left(I-D D^{*} A^{*} P\right)\right]^{-1}=\left\{I+(-A)^{1-\alpha} R(z)(-A)^{\alpha} D^{*} A^{*} P\right\}^{-1}(z I-A)^{-1} .
$$

The brace is bounded for $\Re e z>0$ since $A^{1-\alpha} R(z) o\left(1 /|z|^{\alpha}\right)$. Consequently $\left|R\left(z ; A^{+}\right)\right|<$ $M /|z|$ for $\Re e z>0$ i.e. $A^{+}$generates a holomorphic semigroup.

Now we use again Parseval equality and the fact that $A, A_{+}$generates holomorphic semigroups in order to arrive at the usual Riccati equation. We note that the following computations make sense for $x \in \operatorname{dom} A_{+}, y \in \operatorname{dom} A$ :

$$
\begin{aligned}
& \left\langle y, P A_{+} x\right\rangle=\int_{-\infty}^{+\infty}\left\langle y, R^{*}(i \omega ; A) Q R\left(i \omega ; A_{+}\right) A_{+} x\right\rangle \mathrm{d} \omega \\
& =\int_{0}^{+\infty}\left\langle e^{A t} y, Q e^{A_{+} t} A_{+} x\right\rangle \mathrm{d} t=-\langle y, Q x\rangle-\int_{0}^{+\infty}\left\langle e^{A t} A y, Q e^{A_{+} t} x\right\rangle \mathrm{d} t= \\
& -\langle y, Q x\rangle-\left\langle A y, \int_{0}^{+\infty} e^{A^{*} t} Q e^{A_{+} t} x\right\rangle \mathrm{d} t= \\
& -\langle y, Q x\rangle-\left\langle y, A^{*} \int_{0}^{+\infty} e^{A^{*} t} Q e^{A_{+} t} x\right\rangle \mathrm{d} t=-\langle y, Q x\rangle-\left\langle y, A^{*} P x\right\rangle .
\end{aligned}
$$

Hence we obtained the following equality which holds for each $y \in \operatorname{dom} A$ and $x \in \operatorname{dom} A_{+}$:

$$
\left\langle y, P A\left[I-D D^{*} A^{*} P\right] x\right\rangle=-\langle y, Q x\rangle-\langle A y, P x\rangle
$$

Now, $y \in \operatorname{dom} A$ so that the first inner product is equal to

$$
\left\langle y, P A\left[I-D D^{*} A^{*} P\right] x\right\rangle=\left\langle A^{*} P y, x\right\rangle-\left\langle D^{*} A^{*} P y, D^{*} A^{*} P x\right\rangle .
$$

Hence we arrive at the Riccati equation

$$
\langle y, P A X\rangle+\langle A y, P x\rangle-\left\langle y,\left(D^{*} A^{*} P\right)\left(D^{*} A^{*} P\right) x\right\rangle+\langle y, Q x\rangle=0 .
$$

This equality was derived for any $y \in \operatorname{dom} A$ and any $x \in \operatorname{dom} A_{+}$, but we note that each addendum is a continuous function of $x$ so that the equality can be extended to hold for 
any $x \in X$. In particular, if we choose $x \in \operatorname{dom} A$ we get the usual form of the Riccati Equation.

\section{REFERENCES}

Balakrishnan AV (1978) Boundary control of parabolic equations: L-Q-R theory. in Theory of nonlinear operators. Akademie-Verlag, Berlin.

Bensoussan A., Da Prato G., Delfour M. and Mitter S. (1993) Representation and control of infinite dimensional systems, Birkhäuser, Boston.

Datko, R. (1970) Extending a Theorem of Lyapunov to Hilbert spaces J. Math. Analysis Appl. 32 610-616.

Lasiecka, I., Lukes, D. and Pandolfi, L. (1995) Input dynamics and non-standard Riccati equations with applications to boundary control of damped wave and plate equations, J. Optim. Theory Appl. 84 549-574.

Lasiecka I. and Triggiani R (1991) Differential and algebraic Riccati equations with applications to boundary/point control problems: continuous theory and approximation theory. Springer-Verlag, Berlin. 